УДК 62-503.55

DOI: 10.25140/2411-5363-2020-3(21)-21-36

\author{
Владимир Лебедев
}

\title{
ИСПОЛЬЗОВАНИЕ УПРАВЛЯЕМЫХ ПАРАМЕТРОВ ПОДАЧИ ЭЛЕКТРОДНОЙ ПРОВОЛОКИ И КОЛЕБАНИЙ ВАННЫ КАК ДИНАМИЧЕСКИХ СИСТЕМ ДЛЯ СОВЕРШЕНСТВОВАНИЯ ДУГОВЫХ ПРОЦЕССОВ СВАРКИ И НАПЛАВКИ
}

\begin{abstract}
Актуальность темы исследования. Дуговая сварка и наплавка с применением импульсной подачи электродной проволоки и механических колебаний сварочной ванны являются эффективным средствами существенного повышения качества результатов проведения технологических прочессов, выполняемых механизированным или автоматическим оборудованием. Для рачионального выбора параметров импульсной подачи и колебаний сварочной ванны необходима разработка математического описания результатов применения этих технологий сварки и наплавки.

Постановка проблемы. Для создания методик выбора параметров импульсной подачи электродной проволоки и колебаний сварочной ванны необходимо провести выбор наиболее характерных и применимых форм воздействий, изыскать способы их математического описания и методы решения уравнений, описывающих результаты их действий на технологический процесс.

Анализ последних исследований и публикаций. Наиболее используемыми технико-технологическими приёмами влияния на показатели качества сварного шва и наплавленного слоя, в том числе прочности, является механическое и/или тепловое влияние на сварочную ванну. В последнее время широко применимы такие методы, как периодическое воздействие магнитного поля на расплав сварочной ванны, импульсные режимы сварки и наплавки на базе заданных алгоритмов управления работой оборудования (источники сварочного тока, механизмы подачи электродной проволоки), применение дополнительного легирования наплавляемого металла, а также комбинированные методы.

Выделение неисследованных частей общей проблемы. Несмотря на значительный прогресс в создании новых типов оборудования для сварки и наплавки с импульсными алгоритмами функиионирования, в частности, механизмов подачи с управляемыми параметрами движения до настоящего времени мало исследований, посвящённых математическому описанию действия импульсов различной формы на процессы сварки и наплавки.

Постановка задачи. Провести анализ факторов, влияющих на структуру металла шва и наплавленного слоя с целью повышения качества результатов процессов сварки и наплавки, выявить применяемые формы импульсных воздействий, определить математические описания импульсов, описания динамических проиессов при сварке с учётом движений сварочной ванны. Найти способы решения полученных дифференииальных уравнений.

Изложение основного материала. Показаны результаты импульсных воздействий на структуру металла шва, обеспечивающие существенное улучшение механических свойств сварного соединения и наплавленного слоя. Выбраны и описаны в математической форме импульсы управляемого движения электродной проволоки. Найдены описания проиессов при дуговом воздействии, а также движений сварочной ванны. Получены дифференииальные уравнения импульсных воздействий на результаты сварочного процесса. Решения полученных уравнений основываются на применении операчионного исчисления.
\end{abstract}

Выводы в соответствии со статьёй. Импульсная подача электродной проволоки с управляемыми характеристиками даёт возможность существенно улучшить структуру металла шва, что в значительной степени повьшает прочность сварного соединения и наплавленного слоя, а также снижают энергетические затрать и ресурсопотребление. Технология сварки и наплавки с применением импульсной подачи электродной проволоки наиболее эффективна при рачионально выбранных параметрах импульсного движения проволоки, что невозможно без математического описания этого процесса. Математическое описание в доступном для практического применения виде практически исключает этап достаточно длительных и затратных экспериментальных исследований, а также позволяет прогнозировать ожидаемые результатьл. Дифференциальные уравнения, описывающие импульсные воздействия, целесообразно решать методами теории автоматического регулирования, в частности с использованием операционного исчисления.

Ключевые слова: электродуговая сварка и дуговая наплавка; импульсная подача; анализ; форма импульса; выбор; математическое описание.

Рис.: 1. Библ.: 20.

Актуальность темы исследования. Дуговая сварка и наплавка с использованием плавящегося электрода являются одними из основных в современных технологических процессах при создании новых объектов машиностроения, в строительстве, ремонте и восстановлении уже эксплуатирующихся узлов и деталей. Всё это находит применение при работе в самых различных условиях, в том числе и в водной среде [1] при реализации сварки мокрым способом. Развитие дуговых процессов сварки и наплавки, оборудования для их реализации базируется как на использовании всё более эффективных сварочных материалов, защитных сред, активаторов сварочного процесса, так и на постоянно совершенствующемся оборудовании и применяемым дополнительным системам [2].

(С Лебедев В. О., 2020 
Развиваются и постоянно совершенствуются направления, связанные с импульсными и модулированными алгоритмами работы систем сварочного оборудования, где их выходные характеристики изменяются по заданным параметрам или адаптируются к сварочному или наплавочному процессам, улучшая результаты их работы.

По нашему мнению, именно импульсные воздействия на управление сварочно-наплавочным процессом имеют широкие перспективы и будут развиваться и в дальнейшем, являться основой для новых технико-технологических решений, в том числе с комбинированием воздействий от разных генераторов импульсов.

Постановка проблемы. Целью настоящей работы является рассмотрение и анализ основных тенденций в развитии импульсных техники и технологии сварки-наплавки на основе механизма подачи электродной проволоки с определением направлений их дальнейшего совершенствования с получением аналитических выражений для оценки влияния импульсных воздействий на характеристики металла шва и энергетические параметры процесса, как основных составляющих обеспечивающих качество и экономичность получения сварных швов и наплавленных валиков.

В связи с тем, что теоретическая база по управлению процессом сварки- наплавки с использованием современных источников сварочного тока с импульсным воздействием на перенос электродного металла, в том числе и синергетическим управлением достаточно развита и описана в технической литературе, например [3; 4], в настоящем материале мы будем в большей степени уделять работе систем импульсной подачи электродной проволоки, которые также могут работать в синергетических режимах.

Следует отметить, что основное назначение импульсной системы подачи электродной проволоки это организация управляемого переноса электродного металла, при этом, в зависимости от способа управления переносом могут быть решены следующие основные задачи:

- организация переноса электродного металла с заданными частотными характеристиками (частота, скважность) и обеспечением стабильности процесса;

- управление геометрическими параметрами сварного шва и наплавленного валика.

- снижение энергетических затрат, в том числе за счёт снижения тепловложений в процесс;

- управление процессом формирования структуры металла жидкой ванны за счёт влияния на фронт кристаллизации;

- возможность ведения сварочного и наплавочных процессов с качественным результатом в положениях отличных от горизонтального;

- получение качественных результатов сварки-наплавки на обеих полярностях с использованием их положительных эффектов, в том числе формирование шва и наплавленного валика, глубины проплавления и зоны термического влияния;

- обеспечение снижения доли основного металла в структуре наплавленного упрочняющего наплавленного слоя;

- решение проблем сварки-наплавки для разных металлов (стали, в том числе нержавеющие, чугуна, сплавов алюминия) при использовании оборудования с импульсными выходными характеристиками при этом важный эффект, который можно особо отметить - это возможность качественной сварки особого вида соединений разнородных дуплексных сталей.

Достижение отмеченных результатов важно в создании атомных энергетических установок, корпусных элементов судов и других сложных и ответственных конструкций и сооружений.

Очевидно, что полный выбор всех преимуществ с использованием импульсных алгоритмов управления дуговых процессов одновременно достаточно сложен. Однако ряд эффектов одновременно можно получить, используя возможности настройки режимов и формирования параметров импульсных воздействий. 
При этом полученные, а также ожидаемые результаты основываются на детальном изучении процессов и результатов управления переносом электродного металла, современных достижениях техники, а также на требованиях, предъявляемых техническим прогрессом.

Анализ последних исследований и публикаций. Получение тем или другим способам мелкозернистой первичной микроструктуры металла шва это основной фактор, позволяющий в значительной степени улучшить качество процессов электродуговой сварки и наплавки. В этом случае вредные примеси более равномерно распределяются по границам первичных зерен, снижая, таким образом, склонность к хрупкому разрушению. Уменьшить размеры столбчатых дендритов можно модифицированием, например, путем введения в сварочную ванну наночастиц оксидов титана или циркония [5], а также регулированием процесса кристаллизации, осуществляемом при малом тепловложении. Последнее можно реализовать, если использовать в полной мере возможности инверторных источников питания с синергетическим управлением, а также импульсной подачи электродной проволоки или различных способов механических колебаний сварочной ванны, в том числе и тех, которые изучаются нами.

Следует отметить, что важной особенностью применения импульсных процессов сварки и наплавки нанесение антикоррозионных покрытий, в частности при выполнении ограничений по содержанию $\delta$-феррита, который снижает пластичность и повышает хрупкость наплавленного слоя. Импульсные алгоритмы работы современных источников сварочного тока и систем подачи электродной проволоки обеспечивают снижение тепловложения и степени перемешивания наплавляемого металла с основным, а следовательно, и содержание $\delta$-феррита, что позволяет резко сократить объем наплавленного слоя [6].

В технической литературе рассматриваются вопросы, связанные с дендридной ликвацией в металле сварного шва или наплавленного валика как весьма нежелательного явления [7], при этом отмечены способы борьбы с ним, включая и введение разного вида механических колебаний. Введение таких колебаний может быть реализовано рядом способов, основные из которых это импульсная подача электродной проволоки, колебания свариваемого или наплавляемого изделия [8; 9], введение колебаний дополнительными устройствами [10].

Большая часть результатов, получаемых при импульсной подаче электродной проволоки связана с более интенсивными колебаниями сварочной ванны, производимыми, в том числе силовыми воздействиями дуги.

В работе [11] также рассмотрены некоторые возможности экономии электроэнергии при сварке с импульсными составляющими скорости при подаче электродной проволоки с учётом технических и технологических эффектов и возможных дополнительных затрат.

Следует указать, что непосредственные замеры расхода электроэнергии на плавление электродной проволоки при дуговой сварке со значительными колебаниями тока и напряжения процесса осуществить достаточно сложно. Требуются приборы, учитывающие изменяющийся во времени и по величине характер сварочного тока и напряжения, особенно выраженный при сварке в $\mathrm{CO}_{2}$ с короткими замыканиями дугового промежутка.

Для оценки энергетических затрат при различных видах переноса электродного металла предложено использовать определение электроэнергии, которое необходимо для реализации сварочного процесса. При этом учитывается, что механизированная дуговая сварка осуществляется от источников с жёсткой вольтамперной характеристикой. Для этого разработана система измерения, которая применялась при определении удельных затрат электричества в пяти вариантах переноса электродного металла при сварке в среде $\mathrm{CO}_{2}$ в случаях невозмущённой и импульсной подачи электродной проволоки СВ08Г2С диаметром 1,2 мм. Система подробно рассмотрена в работе. Имеются оригинальные системы измерения, выходным параметром которых является количество электричества, 
затрачиваемое на процесс сварки. Характеристики переноса электродного металла и расходы электроэнергии с учётом адекватности затраченного электричества и электроэнергии приведены в табл. 1.

Таблица 1

Удельные расходы энергии при разных характеристиках и способах сварки

\begin{tabular}{|c|c|c|c|c|c|}
\hline \multirow[b]{2}{*}{$\begin{array}{c}\text { Способ переноса } \\
\text { электродного металла }\end{array}$} & \multirow[b]{2}{*}{$\begin{array}{c}\text { С естественными } \\
\text { короткими } \\
\text { замыканиями }\end{array}$} & \multicolumn{2}{|c|}{$\begin{array}{c}\text { С управляемыми } \\
\text { короткими замыканиями }\end{array}$} & \multicolumn{2}{|c|}{ Без коротких замыканий } \\
\hline & & $\begin{array}{c}\text { Ускорение в } \\
\text { импульсе } \\
\text { м/с }{ }^{2} \\
20-30\end{array}$ & $\begin{array}{c}\text { Ускорение в } \\
\text { импульсе } \\
\text { м/с } 2 \\
40-60\end{array}$ & $\begin{array}{c}\text { Величина } \\
\text { импульса } \\
\text { тока, А, } 50\end{array}$ & $\begin{array}{c}\text { Величина } \\
\text { импульса } \\
\text { тока, A, } 100\end{array}$ \\
\hline $\begin{array}{l}\text { Удельный расход электри- } \\
\text { чества, q пл, } \mathrm{A} \cdot \mathrm{C} / \mathrm{Mм}^{3}\end{array}$ & $2,5-2,7$ & $2,1-2,3$ & $1,9-2,0$ & 1,8 & до 1,6 \\
\hline
\end{tabular}

При исследованиях, проведенных некоторое время тому назад, использовался полуавтомат типа ПШ107И с механизмом импульсной подачи, обеспечивающий механическими устройствами регулируемую форму импульса, позволяющий получить различные ускорения электродной проволоки и выбрать из них необходимые.

В качестве источника сварочного тока выбран простой по конструкции выпрямитель типа ВС300 с регулированием напряжения холостого хода переключением обмоток трансформатора, т.е. с неискажённой формой выходного напряжения, позволяющей исключить влияние формы напряжения на плавление электродной проволоки.

Из табл. 1 видно, что на потребление энергии при сварке существенно влияет способ сварки: при импульсной подаче по сравнению с обычной (невозмущённой подачей) потребление электроэнергии снижается, при этом с увеличением ускорения движения электродной проволоки в импульсе фиксируется снижение потребления электроэнергии. Это частично подтверждено в работах [12; 13], но требует продолжения исследований в этом направлении.

Можно заключить, что параметры импульсной подачи влияют на потребление энергии при импульсном движении электродной проволоки.

Выделение неисследованных частей общей проблемы. Современные способы импульсной подачи характеризуются возможностями управления частотой, скважностью, а также формой и даже возможностью реверсирования направления движения. Такое разнообразие возможностей управления предопределяет поиск математического описания влияния импульсной подачи электродной проволоки на формирование (изменение) характеристик дугового процесса, в частности тока при сварке, наплавке с короткими замыканиями дугового промежутка. Это также относится к изысканию зависимостей влияния импульсной подачи на характеристики движения расплава сварочно-наплавочной ванны, определяющего возможности снижения роста столбчатых кристаллов.

В последнее время создана новая технология дуговой сварки с дозированной подачей электродной проволоки, позволяющая решать целый ряд проблем, возникающих при реализации процессов сварки-наплавки плавящимся электродом. Подробно процесс описан в работе [14]. Благодаря новому набору параметров в ИЭС им. Е. О. Патона создана стандартная технология, обеспечивающая надежную, экономичную и высококачественную сварку с воспроизводимыми результатами и минимально необходимой доработкой сварных изделий. Широкий диапазон регулирования значительно расширяет область применения в отношении толщины материала по сравнению с аналогичными стандартными наборами параметров.

Ранее такая часть работы выполнялась по отношению к выявлению зависимости характеристик импульсной подачи на ток дугового процесса для процесса дуговой сварки плавящимся электродом в защитном газе с короткими замыканиями дугового промежутка [15]. Следует заметить, что в этой работе рассматривался, как теперь определено, только частный 
случай, который относился к возможностям известных в то время механизмов импульсной подачи. К настоящему времени разработаны и реализованы в конструкциях новые системы подачи электродной проволоки, базирующиеся на применении безредукторных механизмов с вентильными электродвигателями с микропроцессорным управлением и регулированием. Такие системы позволят получить импульсное движение электродной проволоки, практически с любыми алгоритмами формирования законов этого движения.

Постановка задачи. Для реализации возможностей наиболее эффективного использования импульсных воздействий на сварочный процесс и непосредственно ванну расплавленного металла необходимо получение математического описания влияния характеристик импульсного движения на ток процесса и движение расплава металла ванны. Влияние скорости подачи электродной проволоки на сварочный ток можно изучать, задавая различные законы движения проволоки.

Изложение основного материала. Для решения этой задачи формализуем формы импульсов подачи. При этом определим две основные применяемые формы: практически синусоидальную (модуль синуса) и приближающуюся к прямоугольной. Такая формализация графически представлена на рис. 1.

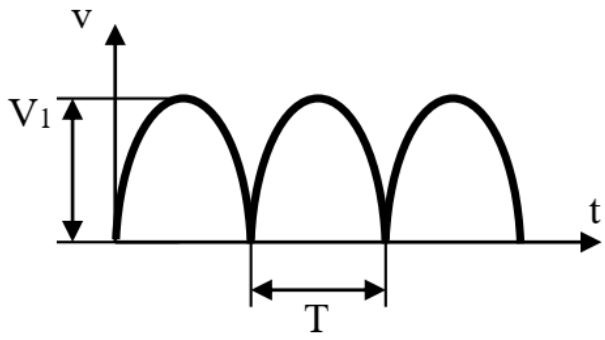

$a$

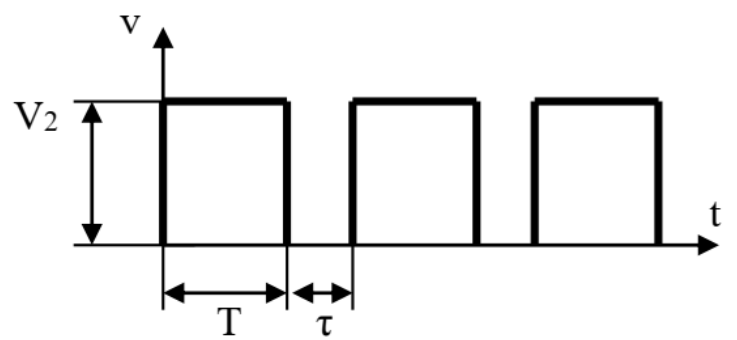

$\sigma$

Рис. 1. Идеализированные формы импульсной подачи электродной проволоки: $а$-синусоидальная, б - приближенная к прямоугольной

Математические описание импульсов подачи электродной проволоки синусоидальной по модулю формы, представленных на рис. 1 будет иметь виды (рис. $1, a$ ).

$$
f_{1}(t)=V_{1}|\sin \omega t| \quad \omega \geq 0
$$

где $V_{l}$ - амплитудное значение скорости импульсной подачи электродной проволоки; $\omega$ - частота следования импульсов (рис. $1, \sigma)$.

$$
f_{2}(t)= \begin{cases}V_{2}, & n T \leq t \leq n T+\tau \\ 0, & n T+\tau \leq t \leq(n+1) T, \quad n=1,2,3 \ldots\end{cases}
$$

где $V_{2}$ - величина прямоугольного импульса; $T, \tau$ - время действия импульса и паузы прямоугольного импульса соответственно.

Существует ряд способов математического описания переходных процессов при различных воздействиях, которые, как правило, базируются на математическом аппарате теории автоматического регулирования. Анализируя эти способы нами выбран, по нашему мнению, наиболее рациональный для тех задач, которые определяют параметры при импульсных влияниях со стороны системы подачи электродной проволоки - это использование операционного исчисления, математический аппарат которого известен и достаточно широко применяется, в том числе, и при исследовании переходных процессов.

Далее рассмотрим математическое описание процессов, происходящих в объекте исследования - дуговом процессе при импульсном воздействии со стороны подачи плавящейся электродной проволоки и сделаем это в двух аспектах: 
- нахождение тока дугового процесса в зависимости от параметров импульсов движения проволоки, что является необходимым, в том числе и для решения задач определения энергетических характеристик процесса сварки при импульсном воздействии со стороны системы подачи;

- нахождение параметров колебаний ванны жидкого металла, зависящих от характеристик импульсов, что определяет характер движения расплава, а в итоге качество металла шва при импульсном воздействии при подаче электродной проволоки.

В цитируемой работе [15] выведено уравнение, связывающее ток дугового процесса и скорость подачи электродной проволоки для дуговой сварки плавящимся электродом в защитном газе с короткими замыканиями дугового промежутка в операторной форме в виде изображения. Используем его в следующем виде

$$
\frac{i(p)}{v(p)}=\frac{\frac{E}{(R+0,5 b)}}{T p^{2}+p+k},
$$

где $i, v$ - текущие значения тока дугового процесса и скорости подачи электродной проволоки соответственно; $p$ - оператор дифференцирования;

$$
T=\frac{L}{R+0,5 b} ; \quad k=\frac{A H E}{R+0,5 b},
$$

где, в свою очередь $L, R$ - индуктивность и эквивалентное сопротивление сварочной цепи соответственно; $b$ - коэффициент, характеризующий наклон статической характеристики дуги к оси токов; $E$ - напряжённость электрического поля в столбе дуги; $A=\frac{1}{M \pi r^{2}} ; H=U_{x x}(0,285-0,0052 U)$ где $U_{x x}-$ напряжение холостого хода источника сварочного тока, а $M=C_{n} \gamma_{n} T_{n}-C_{o} \gamma_{o} T_{o}+r_{o} \gamma_{o}$, где $C_{n}$, $C_{o}$ - теплоёмкость металла при температурах плавления и окружающей среды соответственно; $\gamma_{n}, \gamma_{o}$ - плотность металла при температурах плавления и окружающей среды соответственно; $r_{n}$-скрытая теплота плавления; $T_{n}, T_{o}$ - температура плавления электродного металла и температура окружающей среды соответственно.

В работе [16] показано, что в процессе кристаллизации (в зоне роста дендридов) присутствуют четыре основных физических явления: теплопередача, рост кристаллов, переход примесей, движение жидкого металла, которое можно описать дифференциальным уравнением. При этом получено дифференциальное уравнение динамической системы кристаллизации сварочно-наплавочной ванны в каноническом виде, которое при начальных нулевых условиях и исследовании влияния изменяющихся воздействий системы на кристаллизацию металла, в частности импульсной подачи электродной проволоки, можно представить в виде Лапласова изображения. Опираясь на результаты исследования [17] и учитывая цели настоящей работы запишем несколько преобразованное уравнение, которое связывает скорость импульсной подачи электродной проволоки и движение расплавленного металла в операторной форме, по которому можно судить о степени влияния характеристик импульсов на интенсивность указанного движения, а, следовательно, на рост дендридов кристаллизующего металла в виде изображения:

$$
\frac{v_{\theta}(P)}{v(p)}=\frac{b p+b_{1}}{c_{1} p^{2}+c_{2} p+c_{3}},
$$

где $v_{b}$ - текущее значения колебаний сварочной ванны соответственно 
TECHNICAL SCIENCES AND TECHNOLOGIES

$$
b=B+C=\left(1-k_{0}\right) \frac{c_{0} S_{1} N_{1} \mu_{1}}{l_{0} S_{0}}+C,
$$

где в свою очередь $k_{0}$ - равновесный коэффициент распределения примесей (для стали и углерода $\left.k_{0}=0,46\right) ; c_{0}$ - исходная концентрация примеси в расплаве ванны; $S_{1}, N_{1}$ - коэффициенты описывающие состояние примесей в расплаве; $\mu_{l}-$ коэффициент роста скорости движения фронта кристаллизации от переохлаждения; $l_{0}-$ протяжённость зоны активного роста дендридных зародышей $S_{0}, C$ - состав и концентрация примесей в зоне расплава активного роста дендридных зародышей; $b_{1}-\frac{\mu_{1} \gamma}{l_{0}}$, где $\gamma$ - плотность расплава; $c_{1}=1 ; c_{2}=\frac{k_{m}+k_{d}}{l_{0}}$, где $k_{m}-$ коэффициент массопередачи; $k_{d}=\frac{\left(1-k_{0}\right) \beta_{0} C_{0} s_{1} N_{1} \mu_{1}}{S_{0}}$, где $\beta_{0}$ - коэффициент падения температуры ликвидус в сплаве; $C_{0}-$ коэффициент примеси в жидкой фазе; $c_{3}=\frac{\beta_{0} \mu_{1} \gamma}{l_{0}}$.

В уравнении (4) величины $x_{B x}(p)$ и $x_{\text {выx }}(p)$ обозначают входной и выходной сигналы соответственно. В качестве входных сигналов применяются импульсные воздействия, представленные на рис. 1, с их представлениями, данными в выражениях (1), (2).

Важной является возможность оценки методами теории автоматического регулирования устойчивости систем, описываемых уравнениями (3), (4) при импульсном воздействии со стороны системы подачи электродной проволоки.

Определены раздельно математические описания двух типов входных сигналов для двух видов их влияний на сварочный процесс. Для того чтобы иметь полные математические описания систем входной сигнал - результат его действия, необходимо их привести к единому виду. Это может быть полное использование методов операционного исчисления, тогда сигналы входных воздействий необходимо представить в виде соответствующих изображений. Вторым способом можно воспользоваться, представив сигналы воздействий в удобном для исследований математическом описании и операторные изображения передаточных функций (3), (4) в виде их оригиналов.

Выполним все эти преобразования для определения в дальнейшем наиболее эффективного способа исследования полученных и получаемых уравнений, используя методы теории автоматического регулирования [18]. При этом, как известно, изображение функции периодической последовательности импульсов для примера с длительностью сигналов принятые условно как $\delta$ и периодом $T$ в общем виде и для возможности выбора характеристик импульсных входных воздействий выглядит следующим образом:

$$
F(p)=\frac{1-e^{-\delta p}}{p\left(1-e^{-\delta T}\right)}
$$

Используя функцию Хевисайда

$$
\varphi(t-\delta)=\left\{\begin{array}{l}
0, t \leq \delta \\
1, t \geq \delta
\end{array}\right\}
$$

можно записать, что импульсная ступенчатая функция времени $t$ с периодом $T$ имеет вид: 


$$
f(t)-A-A \phi(t-\delta)=\left\{\begin{array}{l}
A, 0 \leq t \leq \delta \\
0, \delta \leq t \leq T
\end{array}\right\},
$$

где $A=$ consi.

Для периодической последовательности импульсов с длительностью сигналов $\delta$ и периодом $T$ оригинал изображения $F(p)$ можно записать как:

$$
f(t)=A-A \varphi(t-\delta)+A \varphi(t-T)-A(t-T-\theta)+A \varphi(t-2 T-A \varphi(t-2 T-\varphi)+\ldots,
$$

Учитывая периодичность функции $f(t)$ для анализа поведения системы достаточно рассматривать ее значение на протяжении одного периода $T$ следования импульсов. Такое условие обусловлено необходимостью требования технологического процесса импульсной подачи электродной проволоки обеспечить в начале каждого последующего периода следования импульсов нулевые начальные условия работы системы. В противном случае будет нарушено течение сварочного процесса.

Представим выражение (1) для $f_{1}(t)$ используя табличные данные в операторной форме:

$$
V_{1}|\sin \omega t| \leftrightarrow V_{1} \frac{\operatorname{\omega ctg}\left(\frac{\pi p}{2 \omega}\right)}{\left(p^{2}+\omega^{2}\right)}
$$

Выражение (2) для $f_{2}(t)$, при использовании табличных данных в операторной форме примет вид:

$$
\begin{array}{ll}
V_{2}, & n T \leq t \leq n T+\tau \leftrightarrow f_{2}(t) \leftrightarrow \frac{V_{2}\left(1-e^{-p t}\right)}{p\left(1-e^{-p T}\right)} \\
0, & n T+\tau \leq t \leq(n+1) T
\end{array}
$$

Можно записать 4 уравнения, описывающие в операторной форме зависимости между выходными параметрами и входным импульсным воздействием используя полученные уравнения (3) и (4) в выражениях (9) и (10)

1. Обозначив величину $\frac{E}{(R+0,5 b)}=H$, получим выражение в операторной форме, определяющее ток дугового процесса $i(p)$ для импульса по (1)

$$
i_{1}(p)=V_{1} \frac{\omega \operatorname{ctg}\left(\frac{\pi p}{2 \omega}\right)}{\left(p^{2}+\omega^{2}\right)} \frac{H}{T p^{2}+p+k}
$$

2. Для импульса по (2)

$$
i_{2}(p)=\frac{V_{2}\left(1-e^{-p t}\right)}{p\left(1-e^{-p T}\right)} \frac{H}{T p^{2}+p+k}
$$

3. Для определения влияния импульсной подачи электродной проволоки на движение в ванне жидкого металла $v_{1}(p)$ можно для входного импульса по (1) записать

$$
v_{1}(p)=V_{1} \frac{\omega \operatorname{ctg}\left(\frac{\pi p}{2 \omega}\right)}{\left(p^{2}+\omega^{2}\right)} \frac{b p+b_{1}}{c_{1} p^{2}+c_{2} p+c_{3}}
$$

4. Влияние действия импульсной подачи по (2) на характеристики движений в сварочной ванне $v_{2}(p)$ описывается следующим образом: 
TECHNICAL SCIENCES AND TECHNOLOGIES

$$
v_{2}(p)=\frac{V_{2}\left(1-e^{-p t}\right)}{p\left(1-e^{-p T}\right)} \frac{b p+b_{1}}{c_{1} p^{2}+c_{2} p+c_{3}}
$$

Уравнения (11), (12), (13), (14), при их решении дают возможность определить характер переходного тока для тока дугового процесса и движения в жидкой ванне от двух типов импульсных воздействий. Решение этих уравнений, т. е. нахождение оригиналов возможно машинными способами или с использованием упрощений, которые дают разложения в степенные ряды, например, в ряд Тейлора. Применяя такие разложения с несколькими членами можно с достаточными по точности результатами получить изображения входных сигналов в упрощённом виде.

Для (11) получим упрощённое применением степенных рядов Тейлора выражение

$$
\operatorname{ctg}\left(\frac{\pi p}{2 \omega}\right) \approx \frac{1}{\frac{\pi p}{2 \omega}}-\frac{\pi p}{3(2 \omega)} \ldots
$$

Упрощённое выражение для $f_{1}(t)$ будет иметь вид:

$$
f_{1}(t)=V_{1} \frac{2 \omega^{2}\left(\frac{1}{\pi}-\frac{\pi p^{2}}{6 \omega}\right)}{p\left(p^{2}+\omega^{2}\right)} .
$$

Для (10), разложение имеет вид:

$$
\frac{\left(1-e^{-p t}\right)}{p\left(1-e^{-p T}\right)} \approx \frac{1-\left(1-p t+\frac{p^{2} t}{2}\right)}{p\left[1-\left(1-p \tau+\frac{p^{2} \tau}{2}\right)\right]} \ldots
$$

После несложного преобразования упрощённое выражение для $f_{2}(t)$ будет иметь вид

$$
f_{2}(t)=V_{2} \frac{2 t-p}{p(2 \tau-p)} .
$$

Упрощённые математические описания входных импульсов могут быть по аналогии c (11), (12), (13), (14) применены для формирования уравнений, определяющих результат воздействия на процесс сварки с импульсной подачей электродной проволоки с управляемыми параметрами.

Рассмотрим следующий способ исследования влияния импульсной подачи электродной проволоки на характеристики результатов дугового процесса. При этом следует найти обычные выражения для входных сигналов (1) и (2) и оригиналы выражений для изображений (3), (4).

Для входных сигналов по рис. $1, a$, $\sigma$ наиболее рациональный путь - это их представление в виде разложения в ряд Фурье. При этом аппроксимация функции различной формы для импульса по форме (1) в общем случае:

$$
f_{1}(t)=V_{1}\left(\frac{2}{3}-\frac{3}{\pi^{2}} \sum_{n=1}^{\infty} \frac{1-\cos \frac{2 \pi n}{3}}{n^{2}}\right) \cos \frac{2 \pi x n}{3}
$$

Из уравнения (19) возьмём несколько членов, что достаточно для точности наших исследований и после преобразований получим 
TECHNICAL SCIENCES AND TECHNOLOGIES

$$
f_{1}(t)=V_{1}\left[\frac{2}{3}-\frac{3}{\pi^{2}}\left(\frac{3}{2} \cos \frac{2 \pi x}{3}+\frac{3}{8} \cos \frac{4 \pi x}{3}\right)\right]
$$

Для прямоугольного или близкого к нему импульса по форме (2) с использованием общего представления

$$
f_{2}(t)=V_{2}\left[\frac{1}{2}+\frac{2}{\pi} \sum_{k=1}^{\infty} \frac{1}{2 k} \sin \left(\frac{2 k-1}{L} \pi x\right)\right],
$$

Из уравнения (21) возьмём несколько членов, что также достаточно для точно наших исследований и после преобразований получим

$$
f_{2}(t)=V_{2}\left[\frac{1}{2}+\frac{2}{\pi}\left(\frac{1}{2} \sin \left(\frac{1}{L} \pi x\right)+\sin \frac{3 \pi x}{4 L}\right)\right],
$$

где $L$ - период длительности импульса.

Найдём оригинал выражения (3) с указанной выше заменой как $F_{3}(p)$, используя табличные преобразования оригиналов в изображения и выполнив следующие замены и подстановки

$$
\begin{gathered}
\frac{H}{T p^{2}+p+k}=\frac{H}{T\left(p^{2}+\frac{p}{T}+\frac{k}{T}\right)} ; \\
\frac{g}{(p+j)^{2}+g^{2}}=\frac{g}{p^{2}+2 p j+j^{2}+g^{2}} ; \\
2 j=\frac{1}{T} \Rightarrow j=\frac{1}{2 T} ; \\
\frac{k}{T}=j^{2}+g^{2} \Rightarrow g=\sqrt{\frac{k}{T}-j^{2}} \Rightarrow g=\sqrt{\frac{k}{T}-\frac{1}{4 T^{2}}}
\end{gathered}
$$

в результате приведения получим следующее выражение для стандартного вида изображения:

$$
F_{3}(p)=\frac{H}{T p^{2}+p+k}=\frac{H}{T\left(p^{2}+\frac{p}{T}+\frac{k}{T}\right)}=\frac{H}{g T} \frac{g}{(p+j)^{2}+g^{2}} .
$$

Оригинал изображения функции $F_{3}(p)$ будет иметь вид

$$
f_{3}(t)=\frac{H}{g T} e^{-j t} \sin (g t) .
$$

Используя процедуру стандартного приведения изображения функции для выражения (4) в виде $F_{4}(p)$

$$
F_{4}(p)=\frac{b p+b_{1}}{c_{1} p^{2}+c_{2} p+c_{3}}
$$

возможно прийти к стандартному выражению вида

$$
F_{4}(p)=\frac{p+a}{(p+a)^{2}+b^{2}} .
$$

Для использования стандартного выражения выполним подстановку 
TECHNICAL SCIENCES AND TECHNOLOGIES

$$
a=\frac{c_{2}}{2 c_{1}} ; b=\sqrt{\frac{c_{2}}{c_{1}}-\frac{c_{2}^{2}}{4 c_{1}^{2}}} .
$$

Приведём всю цепочку преобразований для получения исходного выражения изображения $F_{4}(p)$ в определении её оригинала $f_{4}(t)$

$$
\begin{gathered}
F_{4}(p)=\frac{b_{1} p+b_{2}}{c_{1} p^{2}+c_{2} p+c_{3}}=\frac{b_{1}}{c_{1}} \frac{p+\frac{c_{2}}{2 c_{1}}}{\left(p+\frac{c_{2}}{2 c_{1}}\right)+\frac{c_{3}}{4 c_{1}^{2}}}+\frac{\left(b_{2}-\frac{c_{2} b_{1}}{2 c_{1}}\right)}{c_{1} \sqrt{\frac{c_{3}}{c_{1}}-\frac{c_{2}^{2}}{4 c_{1}^{2}}} \frac{\sqrt{\frac{c_{3}}{c_{1}}-\frac{c_{2}^{2}}{4 c_{1}^{2}}}}{\left(p+\frac{c_{2}}{2 c_{1}}\right)^{2}+\frac{c_{3}}{c_{1}}-\frac{c_{2}^{2}}{4 c_{1}^{2}}}=} \\
=\frac{b_{1}}{c_{2}} \frac{p+a}{(p+a)^{2}+b^{2}}+\frac{\left(b_{2}-\frac{c_{2} b_{1}}{2 c_{1}}\right)}{c_{1} b} \frac{b}{(p+a)^{2}+b^{2}} .
\end{gathered}
$$

Оригинал изображения (11) функции $F_{4}(p)$

$$
f_{4}(t)=\frac{b_{1}}{c_{1}} e^{-a t} \cos b t+\frac{b_{2}-\frac{c_{2} b_{1}}{2 c_{1}}}{c_{1} b} e^{-a t} \sin b t .
$$

Используя выводы для оригинала можно с некоторым приближением в аналитическом виде найти изменения тока дугового процесса $i_{1}(t)$ при действии импульсов:

по форме (1)

$$
i_{1}(t)=V_{1}\left[\frac{2}{3}-\frac{3}{\pi^{2}}\left(\frac{3}{2} \cos \frac{2 \pi x}{3}+\frac{3}{8} \cos \frac{4 \pi x}{3}\right)\right] \frac{H}{g T} e^{-j t} \sin (g t),
$$

по форме (2)

$$
i_{2}(t)=f_{2}(t)=V_{2}\left[\frac{1}{2}+\frac{2}{\pi}\left(\frac{1}{2} \sin \left(\frac{1}{L} \pi x\right)+\sin \frac{3 \pi x}{4 L}\right)\right] \frac{H}{g T} e^{-j t} \sin (g t) .
$$

C некоторым приближением в аналитическом виде найти изменения скорости колебаний ванны жидкого металла $v_{1}(t)$ при действии импульсов:

по форме (1)

$$
v_{1}(t) V_{1}\left[\frac{2}{3}-\frac{3}{\pi^{2}}\left(\frac{3}{2} \cos \frac{2 \pi x}{3}+\frac{3}{8} \cos \frac{4 \pi x}{3}\right)\right]\left(\frac{b_{1}}{c_{1}} e^{-a t} \cos b t+\frac{b_{2}-\frac{c_{2} b_{1}}{2 c_{1}}}{c_{1} b} e^{-a t} \sin b t\right),
$$

по форме (2)

$$
\begin{gathered}
v_{2}(t) f_{2}(t)=V_{2}\left[\frac{1}{2}+\frac{2}{\pi}\left(\frac{1}{2} \sin \left(\frac{1}{L} \pi x\right)+\sin \frac{3 \pi x}{4 L}\right)\right] \\
\left(\frac{b_{1}}{c_{1}} e^{-a t} \cos b t+\frac{b_{2}-\frac{c_{2} b_{1}}{2 c_{1}}}{c_{1} b} e^{-a t} \sin b t\right) .
\end{gathered}
$$

Для определения оригинала от произведения изображений, как известно, используют теорему о свертке (умножении) их оригиналов [18]. В общем виде выражение свертки оригиналов имеет вид: 


$$
f_{1}(t)^{*} f_{2}(t)=\int_{0}^{t} f_{1}(t-\theta) f_{2}(\theta) d \theta \leftarrow F_{1}(p) F_{2}(p),
$$

где $f_{i}(t), f_{2}(t)$ - функции времени; $F_{1}(p), F_{2}(p)$ - их изображения по Лапласу, соответственно.

При применении теоремы свёртки в нашем случае можно использовать как исходные описания. При использовании исходных описаний получим свёртку для определения переходного процесса по току $i_{i}(t)$ при воздействии по типу (1) в виде

$$
\left.i_{i}(t)=\frac{V_{1} H}{g T} \int_{0}^{t}|\sin \omega(t-\tau)| e^{-j \tau} \sin g \tau\right) d \tau,
$$

где $0<t<\tau$.

Найдём выражение для свёртки при определении переходного процесса по скорости движения ванны $v_{i}(t)$ при воздействии по типу (1) в виде

$$
v_{1}(t)=V_{1} \int_{0}^{t} \mid \sin \omega(t-\tau)\left(\frac{b_{1}}{c_{1}} e^{-\tau a} \cos b \tau+\frac{b_{2}-\frac{c_{2} b_{1}}{2 c_{1}}}{c_{1} b} e^{-a \tau} \sin b \tau\right) d \tau,
$$

где $0<t<\tau$.

Заметим, что выражение для входного сигнала по форме (2) можно записать в виде

$$
f(t)=A-A \varphi(t-\delta) \text {. }
$$

Свёртка для определения переходного процесса по току дугового процесса $i_{2}(t)$ при воздействии по типу (2) в виде

$$
\left.i_{2}(t)=\frac{V_{1} H}{g T} \int_{9}^{t}[A-A \varphi(t-\tau)] e^{-j \tau} \sin g \tau\right) d \tau,
$$

где $0<t<\tau$.

Найдём выражение для свёртки при определении переходного процесса по скорости движения ванны $v_{2}(t)$ при воздействии по типу (2) в виде

$$
v_{2}(t)=\int_{0}^{t}[A-A \varphi(t-\tau)]\left(\frac{b_{1}}{c_{1}} e^{-\tau a} \cos b \tau+\frac{b_{2}-\frac{c_{2} b_{1}}{2 c_{1}}}{c_{1} b} e^{-a \tau} \sin b \tau\right) d \tau .
$$

Интегралы выражений (37), (39), (40) можно при необходимости и интересе к данному исследованию вычислить, используя соответствующие калькуляторы.

Следует отметить, что импульсные процессы сварки и наплавки могут быть использованы не только при сварке и наплавке в среде защитных газов, но и при применении порошковых электродных проволок, а также в других способах дуговой сварки, например $[19 ; 20]$. 
Рассмотренные два способа решения задачи определения зависимостей тока дугового процесса и колебаний сварочной ванны от наиболее применимых форм импульсной подачи электродной проволоки достаточно точны для анализа исследуемых импульсных процессов и результатов их действия.

\section{Выводы в соответствии со статьей.}

Импульсные методы воздействия на процессы дуговой сварки и наплавки плавящимся электродом оказывают значительные эффекты на технологические результаты, способствуя образованию наиболее качественных структур как наплавленного металла, так и околошовной зоны, а также существенно влияют на снижение энергетических затрат, при этом значительных результатов можно достичь, используя импульсные алгоритмы функционирования систем подачи с управляемыми параметрами.

Известные результаты воздействия на различные характеристики дугового процесса импульсных способов подачи электродной проволоки не могут быть таковыми, которые в полной мере описывают исследуемые явления по току и колебаний ванны, так как не позволяют заранее устанавливать параметры импульсного движения электрода без экспериментальных работ, следовательно, необходимы математические описания ожидаемых результатов от задаваемых вариантов импульсов.

Наиболее эффективными способами математического описания воздействий импульсной подачи электродной проволоки являются способы, используемые в математическом аппарате теории автоматического регулирования, в частности операционного исчисления, при этом основой такого исследования являются описания исследуемых фрагментов технологического процесса с их представлением в виде уравнений операционного исчисления, а также описания формализованных импульсов наиболее применяемых форм.

Полученные результаты в виде как приближённых, так и точных уравнений, описывающих исследуемые воздействия с меньшей и большей трудоёмкостью применения, с достаточной точностью для выбора параметров импульсных воздействий для оценки и прогнозирования описывают необходимые для получения качественного результата как по составу метала шва, так и по энергосбережению.

\section{Список использованных источников}

1. Патон Б. Е. Проблемы сварки на рубеже веков. Автоматическая сварка. 1999. № 1. С. 4-14.

2. Бадьянов Б. Н. Некоторые проблемы технологии сварки на рубеже веков. Сварочное производство. 2000. № 1. С. 8-11.

3. Joseph A., Farson D., Harwig D., Richardson R. Influence of GMAW-P current waveforms on heat input and weld bead shape. Science and Technology of Welding and Joining. 2005. Vol 10, No 3. Pp. 311-318.

4. Manikya Kanti K., Srinivasa Rao P., Ranga Janardhana G. Optimization of Weld Bead Penetration in Pulsed Gas Metal Arc Welding using Genetic Algorithm. International Journal of Emerging Technology and Advanced Engineering. 2013. Vol. 3, Issue 3. P. 368-371.

5. Колоколов Е. И., Томилин С. А., Шишов В. В. Обеспечение конструктивной прочности сварных соединений реакторных установок посредством применения новых сварочных материалов и технологий. Глобальная ядерная безопасность. 2017. № 3. С. 77-90.

6. Оборудование и трубопроводы атомных энергетических установок. Сварные соединения и наплавка. Правила контроля. ПНАЭ Г7-010-89. Нормативный документ. Москва : НТЦ ЯРБ, 2000. $164 \mathrm{c}$.

7. Морозов, В. П. Анализ условий формирования измельченной структуры при кристаллизации металла сварочной ванны с наложением внешних периодических возмущений. Известия Высиих Учебных Заведений. Машиностроение. 2006. № 8. С. 41-54.

8. Zhao Y., Lee P.-S., Chung H. Effect of pulsing parameters on drop transfer dynamics and heat transfer behavior in pulsed gas metal arc welding. International Journal of Heat and Mass Transfer. 2019. Vol. 129. Pp. 1110-1122. DOI: 10.1016/j.ijheatmasstransfer.2018.10.037. 
9. Технологические характеристики автоматической наплавки под флюсом с высокочастотными колебаниями электрода / В. А. Лебедев, С. В. Драган, Ж. Г. Голобородько и др. Автоматическая сварка. 2014. № 8. С. 35-38.

10. Lebedev V. A., Solomichuk T. G., Novykov S. V. Study if a Welding Harmonic Oscillation influence on the Welded Metal Hardness and Weld Bead Width. Journal of engineering sciences. 2019. Vol. 6. P. 16-21.

11. Лебедев В. А. Энергосбережении при электродуговой механизированной и автоматической сварке с импульсной подачей электродной проволоки. Автоматизация и современные технологии. 2014. № 1. С. 34-38.

12. Лебедев В. А. Особенности управления процессом сварки плавящимся электродом с импульсной подачей электродной проволоки. Сварка и Диагностика. 2014. № 1. С. 16-18.

13. Лебедев В. А., Жук Г. В. Энергетические затраты при механизированной дуговой сварке и наплавке. Modern question of production and repair in industry and in transport : materials of $18^{\text {th }}$ international scientific and technical seminar (February 10-16, 2018). Brno, Czech Republic, 2018. C. $159-161$.

14. Лебедев В. А., Жук Г. В. Управление переносом электродного метала на основе импульсных алгоритмов функционирования систем с дозированием подачи электродной проволоки при механизированной дуговой сварке. Тяжёлое Машиностроение. 2017. № 6. С. 27-32.

15. Лебедев В. А. Повышение эффективности сварочного оборудования на основе исследования импульсных воздействий в системе подачи электродной проволоки : дис. ... д-ра техн. наук / Институт электростварки им. Е.О. Патона, НАН Украины. Киев, 2010. 327 с.

16. Самойлович Ю. А. Системный анализ кристаллизации слитка. Киев : Наукова думка, 1983. 242 c.

17. Казанский А. А., Игнатьев А. А. Исследование устойчивости фронта дендридной кристаллизации сварочно-наплавочного шва как динамической системы. Вестник СГТУ. 2010 . № 1. C. $48-53$.

18. Бесекерский В. А., Попов Е. П. Теория систем автоматического регулирования. Москва : Наука, 1975.

19. Nadzam J. Tandem GMAW: The Flexibility of Pulsed Spray Transfer. Welding Innovation. 2002. Vol. XIX, No. 2. P. 18-21.

20. Goecke S., Hedegård J., Lundin M. Tandem MIG/MAG Welding. Svetsaren, 2001. No. 2-3. Pp. 24-28.

\section{References}

1. Paton, B. E. (1999). Problemy svarki na rubezhe vekov [Problems of welding at the turn of the century]. Avtomaticheskaia svarka - Automatic welding, 1, 4-14.

2. Badianov, B. N. (2000). Nekotorye problemy tekhnologii svarki na rubezhe vekov [Some problems of welding technology at the turn of the century]. Svarochnoe proizvodstvo - Welding production, 1 , pp. 8-11.

3. Joseph A., Farson D., Harwig D., Richardson R. (2005). Influence of GMAW-P current waveforms on heat input and weld bead shape. Science and Technology of Welding and Joining, 10(3), pp. 311-318.

4. Manikya Kanti, K., Srinivasa, Rao P., Ranga Janardhana, G. (2013). Optimization of Weld Bead Penetra-tion in Pulsed Gas Metal Arc Welding using Genetic Algorithm. International Journal of Emerging Technology and Advanced Engineering, 3(3), pp. 368-371.

5. Kolokolov, E. I., Tomilin, S. A., Shishov, V. V. (2017). Obespechenie konstruktivnoi prochnosti svarnykh soedinenii reaktornykh ustanovok posredstvom primeneniia novykh svarochnykh materialov $\mathrm{i}$ tekhnologii [Ensuring the structural strength of welded joints of reactor plants through the use of new welding materials and technologies]. Globalnaia iadernaia bezolasnost-Global nuclear safety, 3, pp. 77-90.

6. Oborudovanie i truboprovody atomnykh energeticheskikh ustanovok. Svarnye soedineniia i naplavka. Pravila kontrolia. PNAE G7-010-89. Normativnyi dokument [Equipment and pipelines of nuclear power plants. Welded joints and surfacing. Control rules. PNAE G7-010-89. Normative document]. (2000). NTCz YaRB. 
7. Morozov, V. P. (2006). Analiz uslovii formirovaniia izmelchennoi struktury pri kristallizatsii metalla svarochnoi vanny s nalozheniem vneshnikh periodicheskikh vozmushhenii [Analysis of the conditions for the formation of a ground structure during crystallization of the weld pool metal with the imposition of external periodic disturbances]. Izvestiia Vysshikh Uchebnykh Zavedenii. Mashinostroenie - Proceedings of Higher Educational Institutions. Mechanical engineering, 8, pp. 41-54.

8. Zhao, Y., Lee, P.-S., Chung, H. (2019). Effect of pulsing parameters on drop transfer dynamics and heat transfer behavior in pulsed gas metal arc welding. International Journal of Heat and Mass Transfer, 129, pp. 1110-1122. DOI: 10.1016/j.ijheatmasstransfer.2018.10.037.

9. Lebedev, V. A., Dragan, S. V., Goloborodko, Zh. G. et al. (2014). Tekhnologicheskie kharakteristiki avtomaticheskoi naplavki pod fliusom s vysokochastotnymi kolebaniiami elektroda [Technological characteristics of automatic submerged arc surfacing with high-frequency oscillations of the electrode]. Avtomaticheskaia svarka - Automatic welding, 8, pp. 35-38.

10. Lebedev, V. A., Solomichuk, T. G., Novykov, S. V. (2019). Study if a Welding Harmonic Oscillation influ-ence on the Welded Metal Hardness and Weld Bead Width. Journal of engineering sciences, 6, pp. 16-21.

11. Lebedev, V. A. (2014). Energosberezhenii pri elektrodugovoi mekhanizirovannoi i avtomaticheskoi svarke s impulsnoi podachei elektrodnoi provoloki [Energy saving in electric arc mechanized and automatic welding with pulse feed of electrode wire]. Avtomatizatsiia $i$ sovremennye tekhnologii-Automation and modern technologies, 1, pp. 34-38.

12. Lebedev, V. A. (2014). Osobennosti upravleniia protsessom svarki plaviashhimsia elektrodom s impulsnoi podachei elektrodnoi provoloki [Features of control over the process of consumable electrode welding with pulsed electrode wire]. Svarka i Diagnostika - Welding and Diagnostics, 1, pp. 16-18.

13. Lebedev, V. A., Zhuk G. V. Energeticheskie zatraty pri mekhanizirovannoi dugovoi svarke i naplavke [Energy costs in mechanized arc welding and surfacing]. In Modern question of production and repair in industry and in transport: materials of 18th international scientific and technical seminar - Modern question of production and repair in industry and in transport: materials of the 18th international scientific and technical seminar (February 10-16, 2018) (pp. 159-161). Brno, Czech Republic.

14. Lebedev, V. A., Zhuk, G. V. (2017). Upravlenie perenosom elektrodnogo metala na osnove impulsnykh algoritmov funktsionirovaniia sistem s dozirovaniem podachi elektrodnoi provoloki pri mekhanizirovannoi dugovoi svarke [Control of the transfer of electrode metal on the basis of pulsed algorithms for the functioning of systems with dosing of the electrode wire feed in mechanized arc welding]. Tyazhyoloe Mashinostroenie - Heavy Engineering, 6, pp. 27-32.

15. Lebedev, V. A. (2010). Povyshenie effektivnosti svarochnogo oborudovaniia na osnove issledovaniia impulsnykh vozdeistvii v sisteme podachi elektrodnoi provoloki [Improving the efficiency of welding equipment on the basis of the study of impulse effects in the electrode wire feed system] [PhD dissertation, Electric Welding Institute E.O. Paton, NAS of Ukraine].

16. Samojlovich, Yu. A. (1983). Sistemnyi analiz kristallizatsii slitka [System analysis of ingot crystallization]. Naukova dumka.

17. Kazanskii, A. A., Ignatev, A. A. (2010). Issledovanie ustojchivosti fronta dendridnoj kristallizaczii svarochno-naplavochnogo shva kak dinamicheskoj sistemy [Study of the stability of the dendridic crystallization front of the welding-surfacing seam as a dynamic system]. Vestnik SGTU - Bulletin of SSTU, 1, pp. 48-53.

18. Besekerskii, V. A., Popov, E. P. (1975). Teoriia sistem avtomaticheskogo regulirovaniia [Theory of automatic control systems]. Nauka.

19. Nadzam J. (2002). Tandem GMAW: The Flexibility of Pulsed Spray Transfer. Welding Innovation, $X I X(2)$, pp. 18-21.

20. Goecke S., Hedegård J., Lundin M. (2001). Tandem MIG/MAG Welding. Svetsaren, 2-3, pp. 24-28. 
$U D C 4562-503.55$

\section{Vladimir Lebedev}

\section{USE OF CONTROLLED PARAMETERS OF ELECTRODE WIRE SUPPLY AND THE POOL VIBRATION AS DYNAMIC SYSTEMS FOR IMPROVING THE ARC WELDING AND SURFACING PROCESSES}

Urgency of the research. Arc welding and surfacing with the use of pulse feed of electrode wire and mechanical vibrations of the weld pool are effective means of significantly improving the quality of the results of technological processes performed by mechanized or automatic equipment. For a rational choice of the parameters of pulse feed and oscillations of the weld pool, it is necessary to develop a mathematical description of the results of the application of these welding and surfacing technologies.

Target setting. To create methods for choosing the parameters of the pulse feed of the electrode wire and oscillations of the welding pool, it is necessary to select the most characteristic and applicable forms of influences, find ways of their mathematical description and methods for solving equations describing the results of their actions on the technological process.

Actual scientific researches and issues analysis. The most used technical and technological methods of influencing the quality indicators of the weld and deposited layer, including the strength, is the mechanical and / or thermal effect on the weld pool. Recently, methods such as the periodic effect of a magnetic field on the melt of the weld pool, pulsed modes of welding and surfacing based on specified algorithms for controlling the operation of equipment (welding current sources, electrode wire feeders), the use of additional alloying of the deposited metal have been widely applied as well as combined methods.

Uninvestigated parts of general matters defining. Despite significant progress in the creation of new types of equipment for welding and surfacing with pulse algorithms of functioning, in particular, feed mechanisms with controlled motion parameters, there are still few studies devoted to the mathematical description of the effect of pulses of various shapes on the welding and surfacing processes.

The research objective. To analyze the factors affecting the structure of the weld metal and the deposited layer in order to improve the quality of the results of surfacing welding processes, to identify the applied forms of impulse effects, to determine the mathematical descriptions of impulses, descriptions of dynamic processes in welding, taking into account the movements of the weld pool. Find ways to solve the resulting differential equations.

The statement of basic materials. The results of pulsed impacts on the weld metal structure are shown, which provide a significant improvement in the mechanical properties of the welded joint and the deposited layer. Pulses of the controlled motion of the electrode wire are selected and described in mathematical form. The descriptions of the processes under arc action, as well as the movements of the weld pool are found. Differential equations of impulse effects on the results of the welding process are obtained. The solutions of the obtained equations are based on the application of operational calculus.

Conclusions in accordance with the article. Pulse feeding of electrode wire with controlled characteristics makes it possible to significantly improve the structure of the weld metal, which significantly increases the strength of the welded joint and deposited layer, and also reduces energy costs and resource consumption. The technology of welding and surfacing with the use of a pulse feed of an electrode wire is most effective with rationally selected parameters of the pulse movement of the wire, which is impossible without a mathematical description of this process. The mathematical description in a form accessible for practical application practically excludes the stage of rather lengthy and costly experimental research, and also allows predicting the expected results. Differential equations describing impulse influences, it is advisable to solve the methods of the theory of automatic control, in particular, using operational calculus.

Keywords: electric arc welding and arc surfacing; impulse feed; analysis; pulse shape; choice; mathematical description.

Fig.: 1. Table: 1. References: 20.

|Лебедев Владимир Александрович - доктор технических наук, профессор кафедры сварки, Национальный университет кораблестроения имени адмирала Макарова, Херсонский филиал (просп. Ушакова, 44, г. Херсон, 73022, Украина).

Lebediev Volodymyr - Doctor of Technical Sciences, Professor of the Department of Welding, National University of Shipbuilding named by admiral Makarov, Kherson branch (44 Ushakova Av., 73022 Kherson, Ukraine).

E-mail: valpaton@ukr.net

ORCID: https://orcid.org/0000-0003-0391-6113

Лебедев В. Использование управляемых параметров подачи электродной проволоки и колебаний ванны как динамических систем для совершенствования дуговых процессов сварки и наплавки. Технічні науки та технології. 2020. № 3 (21). С. 21-36. 